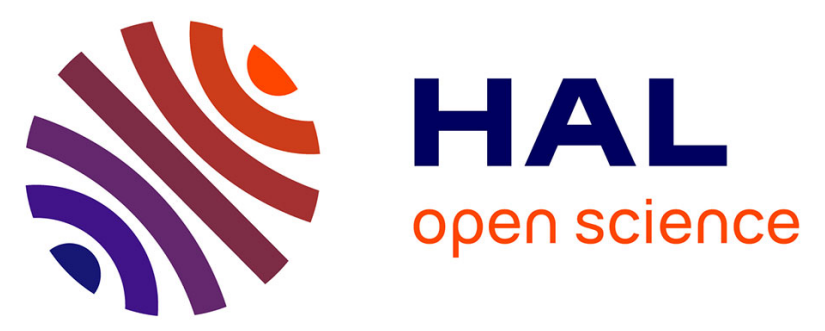

\title{
Crossing of manifolds leads to flat dispersion: Blazed Littrow waveguides
}

\author{
Henri Benisty, Nikolay Piskunov, P.N. Kashkarov, Omer Khayam
}

\section{To cite this version:}

Henri Benisty, Nikolay Piskunov, P.N. Kashkarov, Omer Khayam. Crossing of manifolds leads to flat dispersion: Blazed Littrow waveguides. Physical Review A : Atomic, molecular, and optical physics [1990-2015], 2011, 84 (6), pp.063825. 10.1103/PhysRevA.84.063825 . hal-00683896

\section{HAL Id: hal-00683896 \\ https://hal-iogs.archives-ouvertes.fr/hal-00683896}

Submitted on 19 Nov 2015

HAL is a multi-disciplinary open access archive for the deposit and dissemination of scientific research documents, whether they are published or not. The documents may come from teaching and research institutions in France or abroad, or from public or private research centers.
L'archive ouverte pluridisciplinaire HAL, est destinée au dépôt et à la diffusion de documents scientifiques de niveau recherche, publiés ou non, émanant des établissements d'enseignement et de recherche français ou étrangers, des laboratoires publics ou privés. 


\title{
Crossing of manifolds leads to flat dispersion: Blazed Littrow waveguides
}

\author{
H. Benisty, ${ }^{1, *}$ N. Piskunov, ${ }^{1,2}$ P. K. Kashkarov, ${ }^{2}$ and O. Khayam ${ }^{1}$ \\ ${ }^{1}$ Laboratoire Charles Fabry, Institut d'Optique, Université Paris-Sud, CNRS, FR-91127 Palaiseau, France \\ ${ }^{2}$ Faculty of Physics, M. V. Lomonosov Moscow State University, Moscow, 119991, Russia
}

(Received 23 December 2010; published 12 December 2011)

\begin{abstract}
We display a photonic embodiment of the Demkov-Ostrovsky solution to the crossing of two manifolds made of equidistant modes thanks to broad periodic waveguides. We find clearly narrowing resonances that signal the singular, flat dispersion case that we had termed "critical coupling." The reconciliation of band-edge confinement and cavity confinement, two pillars of photonics, appear from the guide length dependence of spectra. We suggest the generality of the Demkov-Ostrovsky or critical coupling flat dispersion across all kinds of waves, e.g., electronic and acoustic.
\end{abstract}

DOI: 10.1103/PhysRevA.84.063825

PACS number(s): 42.60.Da, 42.82.Et, 71.20.-b, 73.22.Pr

\section{INTRODUCTION}

Systems with a few modes are helpful in relating classical and quantized approaches, for instance, Rydberg states in atom physics. In electromagnetism, tailored low-dimensional photonic structures exhibit Zener tunneling or Landau-Zener transitions [1]. We experimentally exhibit here the modal properties of a broad periodic photonic waveguide (BPW) with three scopes: (i) to show how it addresses a fundamental situation of two manifolds of equidistant states crisscrossing and coupling, solved by Demkov and Ostrovsky in 1995 [2-4]; it is found in Stark manifolds of heavier core-perturbed Rydberg atoms ( $\mathrm{Na}, \mathrm{Rb}, \mathrm{Ca})$ [5-8] or of $\mathrm{H}_{2}$ [9]. The adiabatic manipulation of such atomic manifolds is related to the quantum Galton board [10]. Coherent adiabatic passage techniques have recently been extended thanks to intense laser pulses [11] leading to suggestive analogies [12]. (ii) We reconcile two faces of photonic confinement-cavity confinement and slow light confinement. Our BPW with wavelength-scale period produces slow light extended modes, but these modes also display all the signatures of confined cavity modes. This combination, whereby slow light guides make open cavities, is dual to the popular coupled-resonator-optical-waveguide (CROW) concept [13], in which tunnel-coupled strings of closed cavities make slow light. (iii) We underline the generality of "critical coupling" [14-17], the most singular case of the Demkov-Ostrovsky model, for other guides, whether of electronic [18] or acoustic nature, suggesting numerous applications.

\section{CRISSCROSSING MANIFOLDS, FLAT BANDS, AND CRITICAL COUPLING}

The Demkov-Ostrovsky solution (hereafter DeOsS) to crisscrossing manifolds of constant spacing arose in the context of Stark-split Rydberg states and Landau-Zener (LZ) tunneling. To bridge photonics and atom physics, the diabatic states are those with well-defined hydrogenoid quantum numbers (radial, orbital, magnetic), while extra terms, induced by the ionic core or by dressing, cause Stark-split levels to anticross and form the adiabatic states. LZ theories, known

\footnotetext{
*henri.benisty@institutoptique.fr
}

since 1932, depict how a system evolves, adiabatically or not, when submitted to a growing electric field. In light-wave photonics, the diabatic states are the uncoupled ("bare") states that ignore some periodic dielectric perturbation, the adiabatic states being the eigenstates of the popular coupled mode theory (CMT). Exciting the entrance of a waveguide addresses modes with a restricted set of wave vectors, which can be thought of as a specific combination of the diabatic states, or even as a single state. The exit signal derives from the BPW transmission matrix $T$. It is essentially the Heisenberg evolution $P^{-1} \exp (z D) P$ applied to the entrance excitation $\psi\left(z_{o}\right)$, where $P \equiv|M\rangle\langle\alpha n|$ relates the diabatic $|\alpha n\rangle$ to the adiabatic $|M\rangle$ states $(\alpha= \pm$ relates to forward or backward modes; all states can be labeled by a wave vector $k,|M\rangle=|M(k)\rangle,|\alpha n\rangle=|\alpha n(k)\rangle$ in the first Brillouin zone $|k|<\pi / a$, for a period $a$ ), and $D=E(M, k)|M\rangle\langle M|$ is the diagonalized Hamiltonian $H$ with energies $E(M, k)$. The DeOsS solves an infinite system of equidistant levels, $E_{ \pm}(n, k)=n \Delta E \pm A k$, where the $k$ parameter is formal, being the time $t$ in LZ theory. The coupling term $V$ only couples distinct manifolds, $\left\langle\alpha n|H| \alpha^{\prime} n^{\prime}\right\rangle=0$ if $\alpha \alpha^{\prime}=+1$, and is constant, $\left\langle\alpha n|H| \alpha^{\prime} n^{\prime}\right\rangle=V$ if $\alpha \alpha^{\prime}=-1$.

The most striking feature is what we termed earlier "critical coupling" [15-17], [19,20], the fact that the dispersion $E(M, k)$ can become perfectly flat $(\partial / \partial k \equiv 0)$ for $V_{\text {crit }}=\Delta E / \pi$ [2-4]; see Figs. 1(a) and 1(b). It indeed occurs in limit cases of Stark-split atomic states [5,7,8,21-23]. This property persists well in a truncated $|\alpha n\rangle$ set, or in the case of a distorted spacing, say $n \Delta E \pm A k+\delta E(k)$, because the trend $\langle\alpha n \mid M\rangle \approx 1 /(n-M)$ minimizes the role of far neighbors. The other striking aspect of DeOsS is the reappearance of anticrossing for $V>V_{\text {crit }}$, prompting us to leverage the critical point $V=\Delta E / \pi$ into a general "flat band recipe" in photonic crystal waveguides [15]. This recurrence is part of broader "recurrence spectra" effects such as LZ-Stückelberg interferometry [11,24]. Germane recurrence effects can be tracked in photonics from the Talbot effect to multimode interferometers (MMI) [25] used in every optical node modulator.

We note also that our open waveguide system can be seen as an open resonator comprising a grating. Electromagnetic methods to obtain fields in such open resonators comprising gratings have been proposed many years ago $[26,27]$ and also recently $[28,29]$, but none easily connects to the multimode 


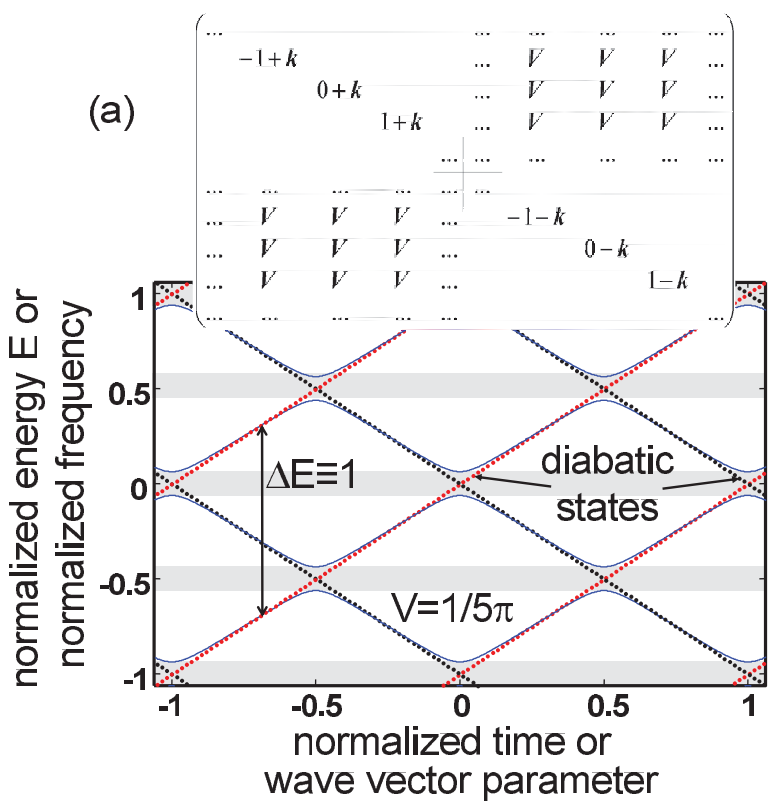

(b)

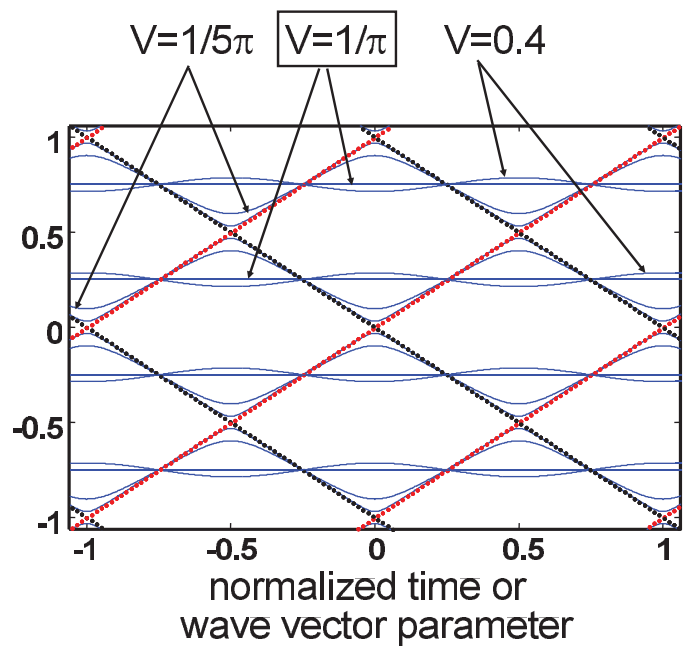

FIG. 1. (Color online) Crossing manifolds of equidistant states (dotted lines) with coupling. The horizontal axis is a parameter that is either a normalized time - in which case the vertical axis is a normalized energy (Landau-Zener situations), or a normalized wave vector-in which case the vertical axis is a normalized frequency (waveguide mode coupling situation). The Demkov-Ostrovsky analytic solution is shown as solid lines: (a) weak coupling and small gaps (horizontal shaded stripes). Top inset: Hamiltonian. (b) Flat dispersion at critical coupling $V=\Delta E / \pi$ and curvature inversion beyond.

coupling approach and Demkov-Ostrovsky correspondence as exploited in the present work.

Let us focus on photonics. Slowdown of light in BPWs was depicted by coupled-mode theory (CMT) $[15,17,19]$. The regime of "collective slow light," called critical coupling regime (CCR), is essentially the flat dispersion at $V=\Delta E / \pi$. Our BPW samples unravel this regime.

\section{BROAD WAVEGUIDE SAMPLES}

Our BPWs have a single corrugated edge. They are etched in a silicon-on-insulator layer of effective index $n_{\text {eff }} \approx 2.83$ at wavelength $\lambda_{o}=1550 \mathrm{~nm}$, processed by EpixFab (IMEC, deep-ultraviolet lithography). Silicon is removed between BPWs [Figs. 2(a) and 2(b)] and between their 3-mm-long $45^{\circ}$ tilted access guides. Higher-order oblique modes in a BPW are of interest when operating around the Littrow grating angle on the grating, in the zigzag ray model (the diabatic states) [14-17,30]. This is also the Brillouin zone edge: The parallel momentum (Bloch wave vector) of these rays obeys $k_{/ /}=$ $\pm \pi / a$. Littman's grating-resonator mount makes use of such incidence, and even of grating pairs [31] but never of parallel ones, so until now, most BPWs were based on photonic crystals $[15,17,19,32,33]$. In a BPW with such $\sim 45^{\circ}$ Littrow angles, only two spatial harmonics $(\alpha= \pm)$ propagate in the core (forward, backward) since $k_{/ /}=\pi / a \sim 0.7 n_{\text {eff }} \omega / c$ is large. The grating acts as a "black box" whose other two-dimensional harmonics can be safely ignored, being evanescent.

Feeding guides end with shallow gratings, to inject transverse electric (TE) polarized light over a $\lambda_{o} \pm 35$-nm range at $2^{\circ}-8^{\circ}$ incident angle. The core design follows a $45^{\circ}$ geometry [16]; see Fig. 2(b). The corrugation of period $a=385 \mathrm{~nm}$ (a)

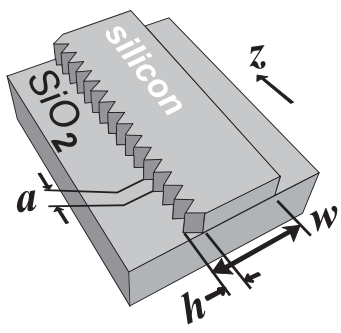

(c)
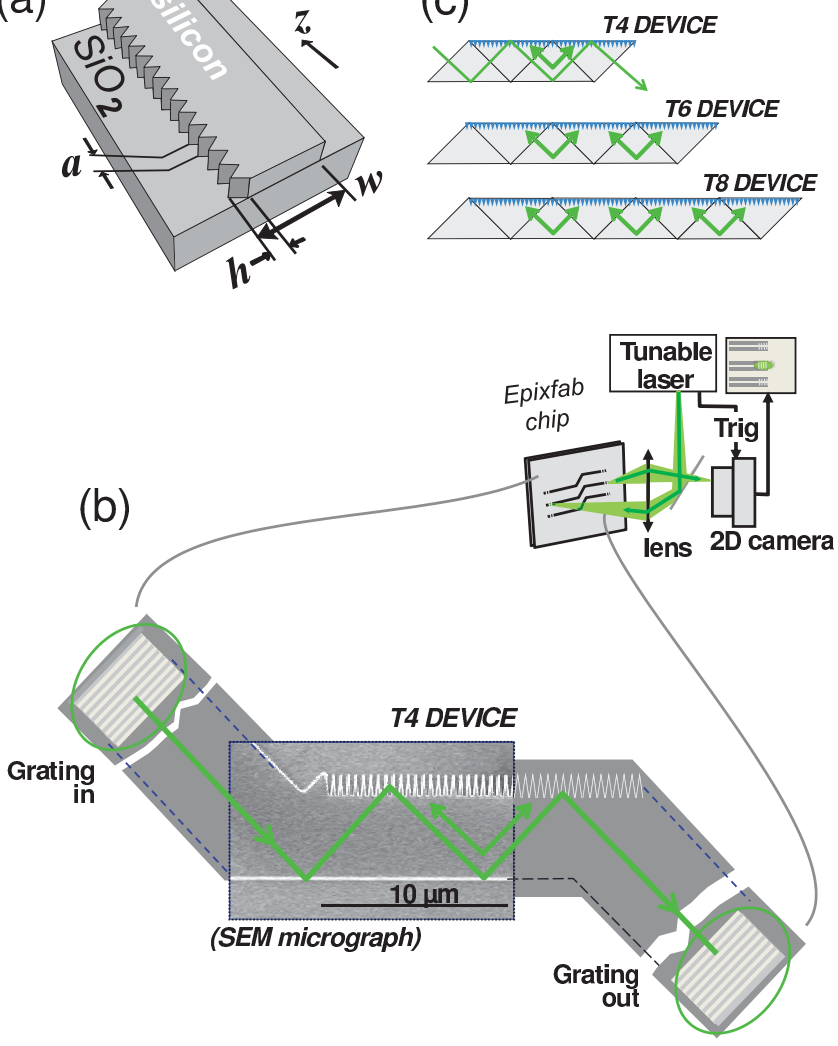

FIG. 2. (Color online) (a) Parameters of a BPW section of order $m$ : width $w=m \lambda / 4 n_{\text {eff }} \sqrt{2}$; corrugation height $h$; period $a$. (b) Composite view of a device with access guides and grating couplers, and schematic resonance; setup is sketched on the right. (c) Length definition of T4, T6, and T8 devices, naturally stemming from the tiling with $4,6,8$ triangles. 
(a)

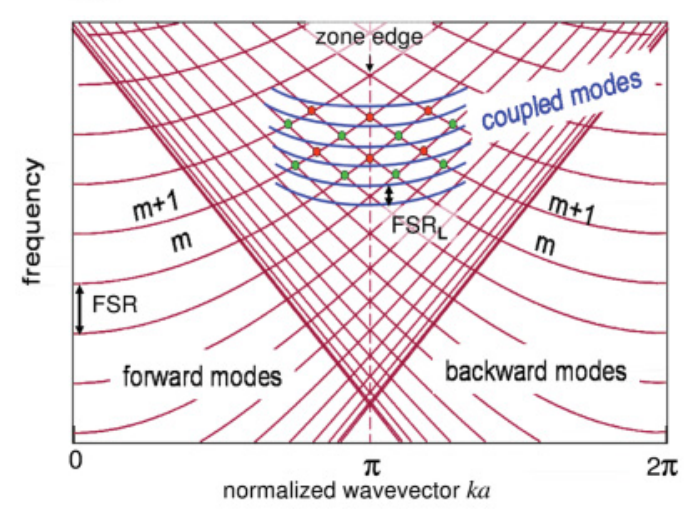

(b)

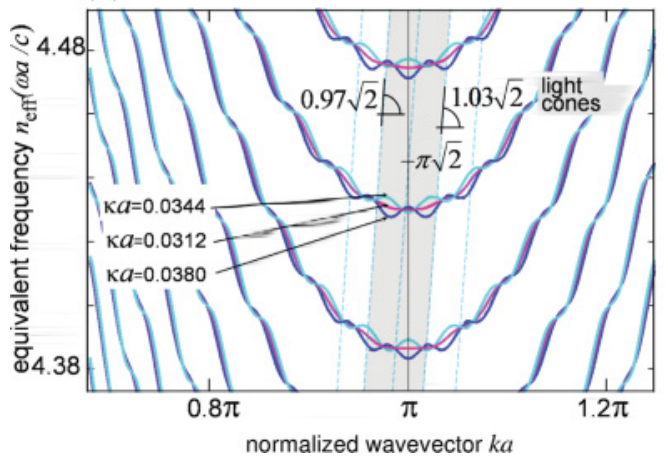

(c)

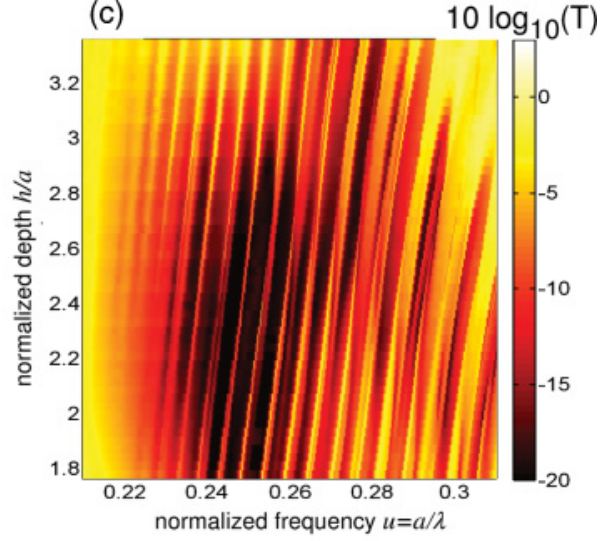

(d)

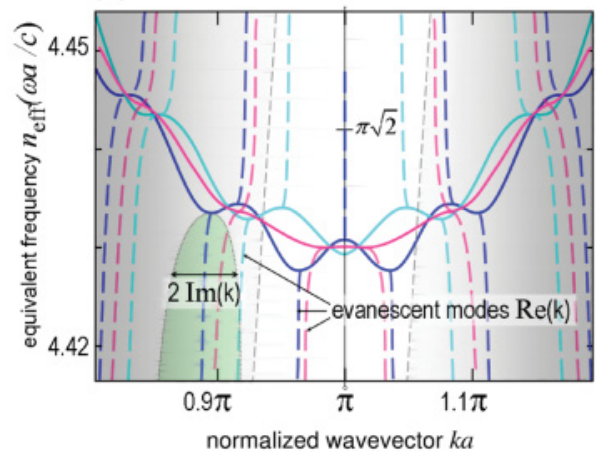

FIG. 3. (Color online) (a) Bands of a broad waveguide (main net of lines) and the DeOsS-type buildup of zone-edge slow-light coupled modes (superimposed segments of hyperbola at top center) upon critical coupling. (b) Detail from CMT, for three coupling constants, chosen below CCR (lighter, wavy line), above CCR (darker, wavy line), and at CCR (the less wavy line); the tilted grey ribbon is the light cone of access guides. (c) Map of simulated T4 device transmission [scale in $\mathrm{dB}$, i.e., $10 \log _{10}(T)$ ] vs frequency and corrugation height; the CCR is the dark area with red stripes. (d) Detail of CCR including evanescent modes (dashed curves) and imaginary dispersion rendered in a typical case (bottom left around $k a=0.9 \pi$ ) by shading the range.

was aimed of height $h$ and of triangular shape [16,17]. For TE polarization, with CCR predicted at $h / a \approx 3.4$, we chose $h / a=$ 2.5-4.0 (the CCR is related to the blaze of the -1 diffraction order $\left.\eta_{-1} \cong 1[15,19,34]\right)$. BPWs are further defined by their width $w$, hence their free spectral range $\mathrm{FSR}_{\mathrm{L}}$ and their Littrow mode order $m=4 n_{\text {eff }} w \sqrt{2} / \lambda$ at design wavelength $\lambda_{o}=1550 \mathrm{~nm}$. We chose $m=50\left(\mathrm{FSR}_{\mathrm{L}} \approx 30 \mathrm{~nm}\right)$ and $m=$ $75\left(\mathrm{FSR}_{\mathrm{L}} \approx 20 \mathrm{~nm}\right)$. Finally, BPW length, Fig. 2(c), is such that a "T $q$ " tiling of $q$ triangles fits a ray-tracing resonator guess [16] and forms $(q / 2-1)$ Fabry-Pérot (FP) in series. Our T4, T6, and T8 sets evoke single, double, and triple FPs.

\section{MODELS AND SIMULATIONS}

Bands of BPWs are well described by CMT [17,19,32]. The (scalar) "diabatic" uncoupled modes are $H_{j}^{ \pm}(x, z) \equiv$ $\sin (j \pi x / w) \exp \left( \pm k_{j} z\right)$ with $k_{j}^{2}+(\pi / w)^{2}=n_{\mathrm{eff}}^{2}(\omega / c)^{2}$. They form two sets of hyperbola in the $(k, \omega)$ plane [Fig. 3(a)]. Couplings around the zone edge $k_{/ /}=\pi / a$ involve modes of opposite slope $\pm v_{g}$. We found earlier that imposing a single coupling strength between all mode pairs, $\kappa_{j, j^{\prime}}=\kappa$, as in DeOsS, still works well $[19,32]$. At CCR, coupled modes evolve into hyperbola centered at $k_{/ /}=\pi / a$ [Fig. 3(a)], essentially the flat DeOsS singular case. Their specific spacing is $\mathrm{FSR}_{\mathrm{L}}=$ $\left(\pi c / 2 n_{\text {eff }} w\right) \cos \theta$, with $\theta=\sin ^{-1}\left(n_{\text {eff }} \omega a / \pi c\right)$, the Littrow angle. The CCR condition in terms of coupling strength is $\kappa=\kappa_{\text {crit }}=v_{g}^{-1} \mathrm{FSR}_{\mathrm{L}} / \pi$ as conjectured in $[17,19,20,32]$ and as shown in the DeOsS.

The resulting flattened bands are shown for three $\kappa$ values [Fig. 3(b)], $\kappa=\kappa_{\text {crit }}=v_{g}^{-1} \mathrm{FSR}_{\mathrm{L}} / \pi, \kappa=0.9 \kappa_{\text {crit }}$, and $1.1 \kappa_{\text {crit }}$. The remaining wiggles stem from two deviations from DeOsS: a "chirped" diabatic level spacing and a semi-infinite set of modes. Many details such as the concavity change are also found in plane wave expansion [17] and are part of blaze singularities [35].

Predictions for finite $\mathrm{T} q \mathrm{BPW}$ of order $m=50$ exploit two-dimensional finite-difference time-domain (FDTD) simulations (CRYSTALWAVE ${ }^{\circledR}$ software; see [36]), using a Gaussian "exciter" at $45^{\circ}$ that mimics a guided mode. The transmission color map $T(a / \lambda, h)$, Fig. 3(c) in $\mathrm{dB}$, uses the reduced frequency $a / \lambda$. To fit measured data, we adjusted the grating pattern. From a high ratio $h^{\mathrm{CCR}} / a \approx 3.4$ for triangles, we realistically got $h^{\mathrm{CCR}} / a \approx 2.5$ (see [36]). The red lines scarring the dark spot around $[a / \lambda \approx 0.25, h / a \approx 2.50]$ correspond to $m \approx 49-51$ resonances and are characteristic of the CCR. A drop in peak transmission $T_{\max }$ occurs along the resonance of a given mode, at variance with ideal FPs $\left(T_{\max } \equiv 1\right.$ in canonical FPs). 

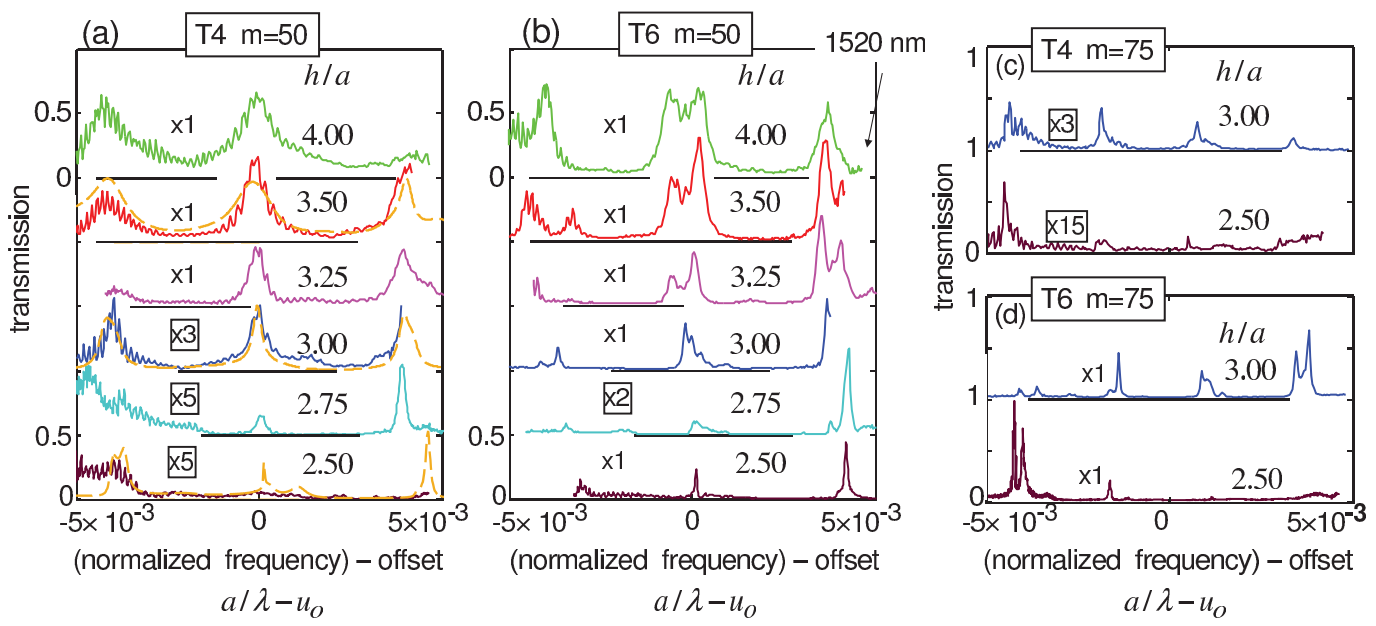

FIG. 4. (Color online) Spectrum of $m=50$ BPW devices of length T4 (a) and T6 (b), with variable $h / a$ ratio. FDTD spectra from Fig. 3 for $h / a \approx 3.50,3.00$, and 2.50 (dashed lines). The measured spectral domain is 1520-1584 nm, but an ad hoc normalized frequency abscissa $u-u_{o}=\left(a / \lambda-u_{o}\right)$ is used to clearly align peaks. (c) Sample of T4 and (d) T6 spectra for $m=75$ and selected $h / a$ values, showing the same poorer peaks for $\mathrm{T} 4$.

Simulations of T6 devices, not shown, display split resonant lines away from CCR, as for two coupled FPs, but a single line is also observed close to CCR. We will address elsewhere the combined role of length and CCR.

\section{RESULTS}

The BPWs are measured by coupling a tunable laser to the sample at an angle through a 50-mm lens. This lens also images light from the exit grating onto an InGaAs array. With acquisitions at each laser scan step $(\Delta \lambda=200 \mathrm{pm}$ or finer), we retrieve the BPW spectrum from the series of raw 14bit images. We sum counts over an area of $3 \times 10$ pixels, discarding spurious light from adjacent guides. A reference straight guide is used for calibration.

Spectra show the predicted trends: For T4 and $m=50$ [Fig. 4(a)], FP peaks with $\mathrm{FSR}_{\mathrm{L}} \approx 30 \mathrm{~nm}$ sharpen up toward the CCR region, much in line with FDTD spectra (dashed lines for three selected heights). This sharpening directly shows that bands in the probed stripe are becoming flat, in line with the DeOsS at $V=\Delta E / \pi$. A drop of $T_{\max }$ is obvious from $h / a=3.00$ to $h / a=2.50$. For T6 [Fig. 4(b)], we see split FP peaks from $h / a=4.00$ to $h / a=3.25$. We see at $h / a=$ 2.75 and 2.50 a recovery of $T_{\max }$ for T6 compared to T4 by a factor of $\sim 10$. The same behavior was observed for $m=$ 75 [Fig. 4(c)]; $h / a=3.00$ and $h / a=2.50$ show a small $T_{\max }$ for T4 and a fairly larger $T_{\max }$ for T6 devices. In longer T8 devices (not shown), we found triplet peaks for $h / a \geqslant 2.75$ (not shown), with quality factors up to $Q \sim 2 \times 10^{4}$, and thus finesses $Q / m 100$, a good value for open resonators.

Two issues, "photonic conductance" [37] and coupling efficiency to slow modes thanks to evanescent modes [38], could be advantageously probed thanks to the underlying analytical DeOsS to get more insight. For instance, Fig. 3(d) shows scarce evanescent modes in our $k$-space window just at CCR.
We have thus evidenced the near attainment of the adiabatic DeOsS "flat band" solution in a photonic context. To perform the converse use of our BPW system in the same spirit as LZ atom manipulations, the wave vector should become the "handle," replacing the Stark shift. One can imagine such a wave-vector control by varying the period during the photon lifetime. It amounts to changing the Brillouin zone edge. But this method is beyond reach for usual solid-state nonlinearities, given our highly contrasted grating indices. Still, the concept could be worked out to produce novel sources based on nonlinear optics. A longitudinal chirp of the period and/or grating strength, for instance, might be thought to push photons traveling down a BPW through controlled states.

The sharp peaks also reveal a strong confinement that does behave as a set of FP cavities. It thus provides FP-type confinement in a strictly wavelength-scale periodic structure, which constitutes a missing link, "dual" of the popular CROW confinement [13]. This reconciliation is useful to assess the merit of nonlinear optical operation for both kinds of confinement.

Our last message is the ease to extend these ideas to other waves, electrons in graphene [18], or acoustical waves. The open nature of the resonator leaves room for any extra longitudinal transport functions. For electrons, an open resonator implements resonant functions through reflections, without any transmission barrier. We generically hope for much less scattering from impurities in the reflection mode than in the transmission mode. As for acoustical open resonators, e.g., in line with $[39,40]$, they could radically alter the noise occurring through receiver or sources. Phonons could also be made resonant the same way in suspended solid nanosystems, for instance, to reach quantum regimes while minimizing the need to thin the holding beams.

\section{CONCLUSIONS}

To conclude, we have evidenced experimentally a generic mode coupling situation that was first addressed in the frame 
of atomic adiabatic Landau-Zener manipulation of Rydberg atoms submitted to Stark splitting, and was solved analytically (DeOsS [2-4]). It predicts the obtainment of flat bands when the coupling potential reaches $V_{\text {crit }}=\Delta E / \pi$. In the framework of multimode broad periodic waveguides, the same phenomenon happens on the band structure $\omega(k)$ at a point that was termed "critical coupling" by us, related to Brillouin zone edge and to the Littman-Metcalf or the Littrow optical mounts. The DeOsS criticality is evidenced here by sharpening transmission spectra when approaching near-unity "blazed" diffraction efficiency, and confirmed by FDTD simulations.
The enriching significance for the canonical classes of photonic confinement (band edge vs cavity), and the general relevance for all kinds of waves (electronic, acoustic, etc.) were underlined.

\section{ACKNOWLEDGMENTS}

The authors thank D. Delande and Dr. Leonid Golovan for useful discussion and Sébastien Benoît, Nicolas François, and Julien Imbert for their help.
[1] S. Longhi, Laser Photonics Rev. 3, 243 (2009).

[2] Y. N. Demkov and V. N. Ostrovsky, J. Phys. B. At. Mol. Opt. Phys. 28, 403 (1995).

[3] Y. N. Demkov, P. B. Kurasov, and V. N. Ostrovsky, J. Phys. A. Math. Gen. 28, 4361 (1995).

[4] V. N. Ostrovsky and H. Nakamura, Phys. Rev. A 58, 4293 (1998).

[5] H. Song and Y. Li, Phys. Rev. A 78, 062504 (2008).

[6] D. A. Harmin, Phys. Rev. A 56, 232 (1997).

[7] N. J. van Druten and H. G. Muller, Phys. Rev. A 52, 3047 (1995).

[8] O. Zobay and G. Alber, Phys. Rev. A 52, 541 (1995).

[9] S. D. Hogan, C. Seiler, and F. Merkt, Phys. Rev. Lett. 103, 123001 (2009).

[10] D. Bouwmeester, I. Marzoli, G. P. Karman, W. Schleich, and J. P. Woerdman, Phys. Rev. A 61, 013410 (1999).

[11] B. Chatel, J. Degert, and B. Girard, Phys. Rev. A 70, 053414 (2004).

[12] D. Bigourd, B. Chatel, W. P. Schleich, and B. Girard, Phys. Rev. Lett. 100, 030202 (2008).

[13] A. Yariv, Y. Xu, R. K. Lee, and A. Scherer, Opt. Lett. 24, 711 (1999).

[14] H. Benisty and O. Khayam, in Proceedings of the 12th International Conference on Transparent Optical Networks (ICTON), (IEEE, Munich, 2010), pp. 1-4.

[15] O. Khayam and H. Benisty, Opt. Express 17, 14634 (2009).

[16] H. Benisty, Photonics and Nanostructures, Fundamentals and Applications 7, 115 (2009).

[17] H. Kurt, H. Benisty, T. Melo, O. Khayam, and C. Cambournac, J. Opt. Soc. Am. B 25, C1 (2008).

[18] H. Benisty, Phys. Rev. B 79, 155409 (2009).

[19] H. Benisty, O. Khayam, and C. Cambournac, Photonics and Nanostructures, Fundamentals and Applications 8, 210 (2010).

[20] H. Benisty, J. Opt. Soc. Am. B 26, 718 (2009).

[21] M. G. Littman, M. L. Zimmerman, T. W. Ducas, R. R. Freeman, and D. Kleppner, Phys. Rev. Lett. 36, 788 (1976).

[22] J. Murray-Krezan, J. Kelly, M. R. Kutteruf, and R. R. Jones, Phys. Rev. A 75, 013401 (2007).
[23] L. Perotti, Phys. Rev. A 73, 053405 (2006).

[24] S. N. Shevchenko, S. Ashhab, and F. Nori, Phys. Rep. 492, 1 (2010).

[25] L. B. Soldano and E. C. M. Pennings, J. Lightwave Technol. 13, 615 (1995).

[26] S. Dowerah and A. Chakrabarti, Indian J. Pure Appl. Math. 16, 411 (1985).

[27] N. V. Veremei and A. I. Nosich, Radiophyics and Quantum Electronics (Engl. Transl.) 32, 166 (1989).

[28] L. G. Velychko and Y. K. Sirenko, Prog. Electromagn. Res. B 16, 85 (2009).

[29] J. P. Zheng and K. Kobayashi, Prog. Electromagn. Res. B 13, 75 (2009).

[30] D. Rosenblatt, A. Sharon, and A. A. Friesem, IEEE J. Quantum Electron. 33, 2038 (1997).

[31] M. G. Littman, Opt. Lett. 3, 138 (1978).

[32] O. Khayam, H. Benisty, and C. Cambournac, Phys. Rev. B 78, 153107 (2008).

[33] O. Khayam, C. Cambournac, H. Benisty, M. Ayre, H. Brenot, G. H. Duan, and W. Pernice, Appl. Phys. Lett. 91, 041111 (2007).

[34] E. Popov, D. Maystre, and G. Tayeb, J. Opt. Soc. Am A 27, 1584 (2010).

[35] F. Schreier, M. Schmitz, and O. Bryngdahl, Opt. Lett. 23, 576 (1998).

[36] See Supplemental Material at http://link.aps.org/supplemental/ 10.1103/PhysRevA.84.063825 for detailed layout of the simulation, and for detailed modification of the grating pattern.

[37] S. Albaladejo, J. J. Saenz, M. Lester, L. S. FroufePérez, and A. Garcia-Martin, Appl. Phys. Lett. 91, 061107 (2007).

[38] T. P. White, L. C. Botten, C. Martijn de Sterke, K. B. Dossou, and R. C. McPhedran, Opt. Lett. 33, 2644 (2008).

[39] J. N. Munday, C. B. Bennett, and W. M. Robertson, J. Acoust. Soc. Am. 112, 1353 (2002).

[40] C. Potel and M. Bruneau, J. Sound. Vib. 313, 738 (2008). 